EPJ Web of Conferences 116, 06001 (2016)

DOI: $10.1051 /$ epjconf/201611606001

(C) Owned by the authors, published by EDP Sciences, 2016

\title{
A Precision Optical Calibration Module (POCAM) for IceCube-Gen2
}

\author{
M. Jurkoviča ${ }^{a}$ K. Abraham, K. Holzapfel, K. Krings, E. Resconi, and J. Veenkamp for the \\ IceCube-Gen2 Collaboration ${ }^{\mathrm{b}}$ \\ Physik-Department, Technische Universität München, James-Franck-Str. 1, 85748 Garching, Germany
}

\begin{abstract}
We present here a new concept of an in-situ self-calibrated isotropic light source for the future IceCube-Gen 2 neutrino detector called the Precision Optical Calibration Module (POCAM). IceCube-Gen2 will be a matrix of light sensors buried deep in the ice at the geographic South Pole. The timing, the location, and the amount of Cherenkov light deposited by the secondary charged particles are used to reconstruct the properties of the incident neutrinos. The reconstruction relies on a detailed detector model that includes the response of optical modules to the Cherenkov light, as well as the optical properties of the detector medium - the natural Antarctic ice. To understand these properties, both natural, and artificial light sources are already used for calibration. New calibration devices are being developed in order to improve the precision of these measurements, and reduce systematic errors. The POCAM concept is based on the principle of an inverted integrating sphere. The main components are LEDs emitting light at several wavelengths and solid-state light sensors e.g. calibrated photodiode or silicon photomultipliers to monitor the emitted light intensity. We report on the current status of the POCAM R\&D.
\end{abstract}

\section{Motivation}

Building on the success of the IceCube detector, a next generation detector is under design, the IceCubeGen2 facility [1, 2]. Two new detectors are under consideration: the Precision IceCube Next Generation Upgrade (PINGU) [3, 4] for determining the neutrino mass ordering and the IceCube-Gen2 high-energy array [5] for high-statistics observation of astrophysical neutrino sources. Both in-ice detector concepts share a common Digital Optical Module (DOM) design including a calibration system similar to that of the current detector, where all DOMs include LED flashers. This system has been used in IceCube to measure ice properties, DOMs' sensitivity, timing, orientation, and position after deployment. It has provided a level of precision required for the present operation but it lacks an in-situ monitoring of the emitted light. LED-to-LED variations, individual DOM sensitivity variations and the optical properties of the refrozen hole ice are the main factors limiting the precision currently obtained in IceCube. An improved calibration system is needed to enable a better understanding of the optical properties of the deep ice and thus significantly reduce systematic effects. We propose here a new calibration device called the Precision Optical CAlibration Module (POCAM).

\footnotetext{
a e-mail: martin.jurkovic@tum.de

b http://icecube.wisc.edu
}

This is an Open Access article distributed under the terms of the Creative Commons Attribution License 4.0, which permits unrestricted use, distribution, and reproduction in any medium, provided the original work is properly cited. 


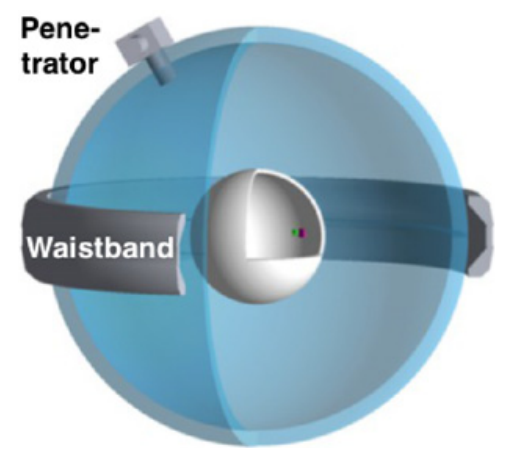

Figure 1. POCAM's baseline geometry consisting of a semi-transparent integrating sphere placed in the middle of the IceCube pressure sphere.

\section{POCAM design and performance goals}

The POCAM, shown schematically in Fig. 1, will be a self-calibrated homogeneous calibration light source. With the POCAM we aim to verify the energy scale, and measure the energy resolution at the few percent level. Here the energy scale verification means the comparison of the expected number of photoelectrons (NPEs) to the NPEs measured by all DOMs' PMTs. Additionally, an even more precise understanding of the optical ice properties and DOM sensitivity will be provided.

In both in-ice detector designs, the POCAM will be located on the communication/power cable alongside the DOMs. For the IceCube-Gen2 high-energy array, POCAM's requirement on the homogeneity can be weakened as compared to PINGU because the inter-string distance on the order of 200-300 $\mathrm{m}$ is much larger than the effective scattering length of up to $\sim 70 \mathrm{~m}$ in the clearest ice [6]. Thus, photons emitted by a bright source will undergo several scattering processes before reaching DOMs at nearby strings. For PINGU, the distance between strings will be $22 \mathrm{~m}$ only. This distance is comparable or even smaller than the effective scattering length in the ice below the dust layer [6]. Therefore, photons will propagate mostly directly from the source to the receiving DOMs at the closest strings. Given the detector symmetry (Fig. 2) in the azimuthal direction and the POCAM's random orientation along the string axes after deployment, we aim for a homogeneous ice illumination on the order of $1 \%$ in the azimuth direction. Because the PINGU baseline geometry has an up-down asymmetry due to the DOMs' asymmetric construction, with a single downward-facing PMT in the lower hemisphere, we allow an inhomogeneity in illumination in zenith direction on the order of $10 \%$.

\subsection{POCAM components}

The POCAM (Fig. 1) is a homogeneous, intense, and fast light source incorporating an in-situ light monitoring system, placed in the IceCube pressure sphere, a housing that will allow POCAM's safe deployment and operation in the deep Antarctic ice. As a light source for the POCAM we consider a matrix of monochromatic fast-switched LEDs driven by the Kapustinsky circuit [7] covering a wavelength range $370<\lambda / \mathrm{nm}<500$. Alternatively, the feasibility of using laser diodes will be investigated, which have become affordable in recent years. The light emitted by a discrete light source will be reflected on the inner wall of a diffusing sphere made from a highly reflective polytetrafluorethylene (PTFE) [8] to generate a homogeneous ice illumination. For the construction of the diffusing sphere we consider two options: (a) a multi-port configuration made of a PTFE with wall thickness $>1 \mathrm{~cm}$ having equidistantly distributed holes (ports) through which photons can escape into the ice and (b) a semi-transparent configuration having a wall thickness of $\sim 2 \mathrm{~mm}$ allowing photons to 


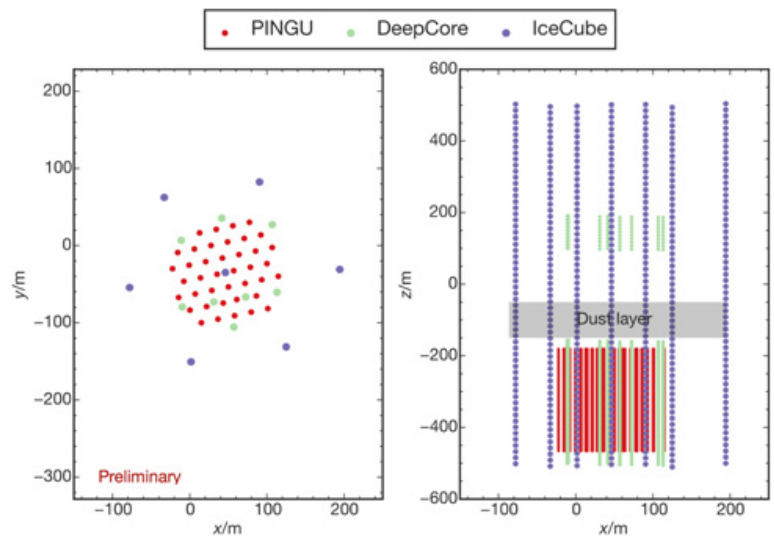

Figure 2. Top (left) and side (right) view of the PINGU baseline geometry showing the position of IceCube, DeepCore, and planned PINGU strings. The $z=0 \mathrm{~m}$ position coresponds to a depth of $1948.07 \mathrm{~m}$ below the surface.

Table 1. POCAM configurations: integrating sphere diameter $D$, number of ports $n$, port opening angle $\alpha$, effective photon transparency $T$, photon absorption $A$, inhomogeneity for three different zenith angle zones and decay constant $\tau$. Configurations $C 3$ and $C 4$ correspond to the semi-transparent setup.

\begin{tabular}{|c|c|c|c|c|c|c|c|c|c|}
\hline \multirow[t]{2}{*}{ Config. } & \multirow[t]{2}{*}{$D / \mathrm{cm}$} & \multirow[t]{2}{*}{$n$} & \multirow[t]{2}{*}{$\alpha /^{\circ}$} & \multirow[t]{2}{*}{$T$} & \multirow[t]{2}{*}{$A$} & \multicolumn{3}{|c|}{ Inhomogeneity } & \multirow[t]{2}{*}{$\tau / \mathrm{ns}$} \\
\hline & & & & & & $\theta<60^{\circ}$ & $60^{\circ} \leq \theta \leq 120^{\circ}$ & $\theta>120^{\circ}$ & \\
\hline C1 & 24 & 768 & 1 & $1.46 \%$ & $61.0 \%$ & $11.9 \%$ & $18.5 \%$ & $11.6 \%$ & 26.4 \\
\hline $\mathrm{C} 2$ & 6 & 768 & 1 & $1.46 \%$ & $74.4 \%$ & $14.7 \%$ & $56.4 \%$ & $10.3 \%$ & 8.1 \\
\hline $\mathrm{C} 3$ & 24 & - & - & - & $50.3 \%$ & $12.4 \%$ & $14.5 \%$ & $12.4 \%$ & 13.1 \\
\hline $\mathrm{C} 4$ & 6 & - & - & - & $50.7 \%$ & $14.3 \%$ & $61.3 \%$ & $10.3 \%$ & 3.1 \\
\hline
\end{tabular}

escape into the ice through the wall with a probability of $\sim 2.5 \%$. The results of the integrating sphere geometry optimization are summarized in the section 3, and more details can be found in [9]. For the continuous in-situ monitoring of the emitted light intensity with a precision of about $1 \%$ we are going to implement fast solid state detectors such as photodiodes or silicon photomultipliers. For the housing of the POCAM the well-tested IceCube pressure sphere is used. This choice reduces the homogeneity of the POCAM via the presence of the penetrator, the waistband/harness, and the cable shadowing. It enables an easy integration of the POCAM into the standard deployment operation.

\section{Results}

To optimize POCAM's geometry we implemented a dedicated Geant4 [10] simulation, for details see [9]. Here we report preliminary results on homogeneity and timing, comparing the multi-port and semi-transparent configuration of the integrating spheres with 6 and $24 \mathrm{~cm}$ diameters, see Table 1 . Properties of all photons leaving POCAM were recorded in $20 \mathrm{~cm}$ distance from POCAM's centre. The average photon emission direction in azimuth direction for different zenith angle bands $\theta$ with $\Delta \theta=1^{\circ}$ is shown in Fig. 3 (left) together with the time profile of the photon emission (right). All configurations show pronounced decrease in photon density at zenith angles around $\theta=30^{\circ}$ and $\theta=90^{\circ}$ caused by the shadow of the penetrator and the waistband. Generally, these shadows get smaller but more pronounced for the configurations with smaller integrating spheres. In the regions without any shadow, e.g. for $\theta>120^{\circ}$, the overall inhomogeneity goes down to $10.3 \%$ for configurations $C 2$ and $C 4$. In narrow zenith bands the semi-transparent configuration $C 4$ has the smallest inhomogeneity on the order of $1.8 \%$. This configuration shows in our simulation not only the most homogeneous illumination, but also the fastest time response with a decay constant $\tau_{4}=3.1 \mathrm{~ns}$. 


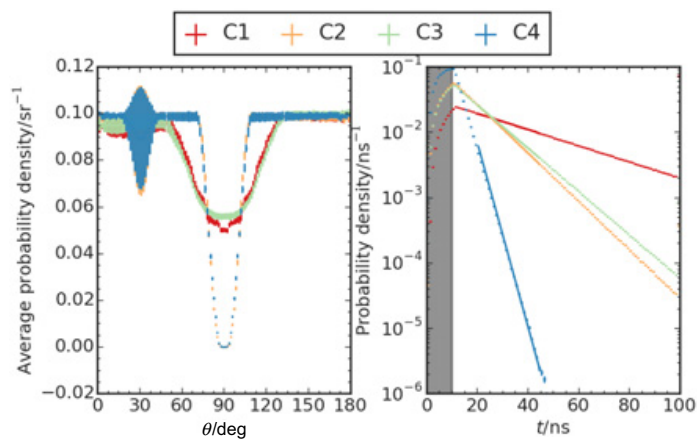

Figure 3. Left: average photon emission direction in azimuth direction for different zenith angle bands. The error bars show the standard deviation. Right: emission time profile with an exponential distribution fitted to each profile for $t>20 \mathrm{~ns}$ (solid line). The shaded area indicates the rectangular LED time profile.

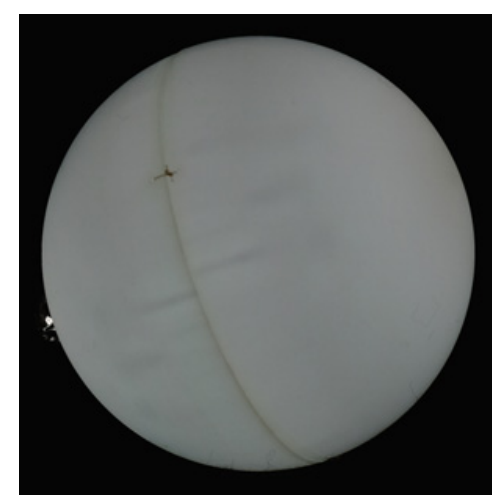

Figure 4. First prototype of the semi-transparent integrating sphere for the POCAM manufactured from commercially available PTFE with $2 \mathrm{~mm}$ wall thickness.

Based on our simulation results, the semi-transparent configuration with $6 \mathrm{~cm}$ diameter is our new baseline geometry for prototyping the integrating sphere for the POCAM. It is shown in Fig. 4 and consists of two hemispheres held together by thin strings. The hemispheres with a wall thickness of $2 \mathrm{~mm}$ were machined out of a massive block of commercially available PTFE. A white LED illuminates the interior of the prototype through a small hole at the pole of the left hemisphere (bright spot). Already by eye, several dark spots close to the equator are clearly visible. All hemispheres produced in this batch show the same pattern. These defects could be explained by the material itself or by the material stress during the machining. For the final prototype we are going to use an optical PTFE-based material from SphereOptics [8]. Nevertheless, this prototype can be used to test and optimize our setup for measuring the light emission from the integrating spheres and from the POCAM.

\section{Conclusions and outlook}

The Precision Optical Calibration Module (POCAM) is a new isotropic light source featuring in-situ light intensity monitoring being developed for the verification of the energy scale and resolution in IceCube-Gen2. With a dedicated Geant 4 simulation we were able to demonstrate that a semi-transparent configuration of the integrating sphere for the POCAM is favored over a multi-port configuration allowing slightly more homogeneous ice illumination (1.8\% inhomogeneity in a narrow zenith band) 
and shorter light pulses with $\tau=3.1 \mathrm{~ns}$. Following this result we build a first prototype of the integrating sphere from a commercially available PTFE with a wall thickness of $2 \mathrm{~mm}$.

Finally, while the POCAM is being developed for IceCube-Gen2, it is a calibration device that has potential use more broadly in the neutrino astronomy community.

\section{References}

[1] K. Hanson (IceCube-Gen2), in VLVnT Workshop, Rome (2015)

[2] M. Aartsen et al. (IceCube-Gen2) (2014), 1412.5106

[3] M. Aartsen et al. (IceCube-PINGU) (2014), 1401.2046

[4] K. Clark (IceCube-Gen2), in International Cosmic Ray Conference (2015)

[5] E. Blaufuss (IceCube-Gen2), in International Cosmic Ray Conference (2015)

[6] D. Chirkin (IceCube), in International Cosmic Ray Conference (2013), 1309. 7010

[7] J. Kapustinsky et al., Nucl. Instrum. Meth. A241(2-3), 612 (1985)

[8] Zenith Polymer ${ }^{\circledR}$ from SphereOptics (www.sphereoptics.de)

[9] K. Krings (IceCube-Gen2), in International Cosmic Ray Conference (2015)

[10] S. Agostinelli et al. (GEANT4), Nucl. Instrum. Meth. A506, 250 (2003) 